\title{
BMJ Open Cost of elective percutaneous coronary intervention in Malaysia: a multicentre cross-sectional costing study
}

\author{
Kun Yun Lee, ${ }^{1}$ Tiong Kiam Ong, ${ }^{2}$ Ee Vien Low, ${ }^{3}$ Siow Yen Liow, ${ }^{4}$ Lawrence Anchah, ${ }^{5}$ \\ Syuhada Hamzah, ${ }^{6}$ Houng Bang Liew, ${ }^{7}$ Rosli Mohd Ali, ${ }^{8}$ Omar Ismail, ${ }^{9}$ \\ Wan Azman Wan Ahmad, ${ }^{10}$ Mas Ayu Said, ${ }^{11}$ Maznah Dahlui ${ }^{1}$
}

To cite: Lee KY, Ong TK, Low EV, et al. Cost of elective percutaneous coronary intervention in Malaysia: a multicentre cross-sectional costing study. BMJ Open 2017;7:e014307. doi:10.1136/ bmjopen-2016-014307

- Prepublication history and additional material areavailable. To view these files please visit the journal online (http://dx.doi. org/10.1136/bmjopen-2016014307).

Received 16 September 2016 Revised 6 April 2017 Accepted 19 April 2017

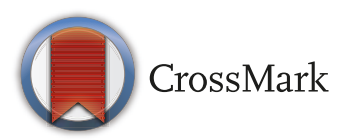

For numbered affiliations see end of article.

Correspondence to

Dr Kun Yun Lee; leekunyun@ gmail.com

\section{ABSTRACT}

Objectives Limitations in the quality and access of cost data from low-income and middle-income countries constrain the implementation of economic evaluations. With the increasing prevalence of coronary artery disease in Malaysia, cost information is vital for cardiac service expansion. We aim to calculate the hospitalisation cost of percutaneous coronary intervention ( $\mathrm{PCl}$ ), using a data collection method customised to local setting of limited data availability.

Design This is a cross-sectional costing study from the perspective of healthcare providers, using top-down approach, from January to June 2014. Cost items under each unit of analysis involved in the provision of $\mathrm{PCl}$ service were identified, valuated and calculated to produce unit cost estimates.

Setting Five public cardiac centres participated. All the centres provide full-fledged cardiology services. They are also the tertiary referral centres of their respective regions.

Participants The cost was calculated for elective PCl procedure in each centre. PCl conducted for urgent/ emergent indication or for patients with shock and haemodynamic instability were excluded.

Primary and secondary outcome measures The outcome measures of interest were the unit costs at the two units of analysis, namely cardiac ward admission and cardiac catheterisation utilisation, which made up the total hospitalisation cost.

Results The average hospitalisation cost ranged between RM11 471 (US\$3186) and RM14 465 (US\$4018). PCl consumables were the dominant cost item at all centres. The centre with daycare establishment recorded the lowest admission cost and total hospitalisation cost.

Conclusions Comprehensive results from all centres enable comparison at the levels of cost items, unit of analysis and total costs. This generates important information on cost variations between centres, thus providing valuable guidance for service planning. Alternative procurement practices for $\mathrm{PCI}$ consumables may deliver cost reduction. For countries with limited data availability, costing method tailored based on country setting can be used for the purpose of economic evaluations.

Registration Malaysian MOH Medical Research and Ethics Committee (ID: NMRR-13-1403-18234 IIR).

\section{Strengths and limitations of this study}

- A multicentre costing analysis using standardised collection methods can lead to within-centre and between-centre comparison at multiple levels, from cost items, units of analysis to overall hospitalisation cost.

- The non-participation of private cardiac centres may limit the generalisability of the results.

- Top-down costing approach applied in this study produced an average estimate cost per patient and enabled an objective comparison of resource consumption and hospitalisation cost between different centres.

- However, this average cost estimates are insufficient for in-depth analysis at patient level or determination of cost predictors via regression analysis.

- This alternative costing methods we devised to overcome the data limitations in our setting can be adapted by like-minded researchers in other lowincome and middle-income countries.

\section{INTRODUCTION}

Economic evaluation is an important component of healthcare delivery. With the increasing prevalence of diseases and advancement of medical technologies, healthcare costs continue to escalate. In high-income countries (HIC) with an easy access to highquality and freely available data, there are well-established guidelines for healthcare economic evaluation. ${ }^{1}$ Therefore, costing analysis of healthcare services are commonly applied to assess the impact of investments in disease prevention and treatment and to guide decision making in budget allocation and service planning. ${ }^{2}$ In contrast, the lack of infrastructure and financial support for evidentiary data capture in low-income and middle-income countries (LMICs) results in a paucity of easily accessible and reliable cost data. This becomes a huge challenge to the healthcare providers in LMIC towards the 
uptake of research and application of economic evaluation. ${ }^{3-6}$

Cardiovascular disease (CVD) is a leading cause of morbidity and mortality in Malaysia. In 2013, CVD accounted for $24.71 \%$ of total mortality and was one of the top five causes of hospitalisation. ${ }^{7}$ CVD encompasses a range of conditions including coronary artery disease (CAD), cerebrovascular disease, peripheral artery disease and heart failure. Among all, CAD accounts for the highest prevalence and mortality. Percutaneous coronary intervention (PCI) is a common treatment modality for CAD due to its safety profile in terms of lower mortality and complications. ${ }^{89}$ In many countries, the availability of coronary catheterisation facilities and access to PCI is a key performance indicator of the healthcare service. However, the need for sophisticated laboratory, highly skilled clinical staff and costly consumables such as cardiac stents often drive up the cost of PCI. This can be a constraining factor towards its service establishment and delivery in resource-limited countries.

Currently, more than 60 public, private and teaching institutions, most of which are tertiary-level referral hospitals located in urban areas, are performing approximately 12000 PCIs annually in Malaysia. This service is not available in district hospitals of lower tiers. In the public healthcare system, cardiology services are provided by teaching hospitals and state-level Ministry of Health hospitals. In addition, Malaysia has a highly specialised national heart institute, which was established as a corporatised entity, and given autonomy in terms of financial and staff autonomy, even though it is fully owned by the government. Cost of medical procedures and hospitalisation often depend on the type of hospitals. International studies have reported cost differences between publicowned or private-owned hospitals, teaching or non-teaching institutions. ${ }^{10} 11$ However, local evidence are lacking, as the development of health economics is still in its infancy stage in Malaysia, and efforts to conduct economic evaluations are hampered by limited access to financial data and non-computerised patient clinical data. Policy makers and healthcare professionals will be interested to gain insight into the local cost variation. In order to do so, reliable cost data from cardiac centres are needed.

\section{OBJECTIVES}

With these in mind, we describe a simple, standardised data collection method for a multicentre costing study of cardiac service in Malaysia. Elective PCI is the chosen procedure as it is among the most commonly conducted cardiac procedure with the least heterogeneity among the patients and providers. We aim to outline the modifications made to existing guidelines. By presenting the cost results from five cardiac centres, we hope to ascertain the cost variation between centres and to identify potential cost-saving options.

\section{METHODS}

\section{Study design}

This costing analysis was designed as a hospital-based cross-sectional study, from the perspective of healthcare providers. Cost data collection was conducted from 1 January 2014 to 30 June 2014, using a top-down costing approach. All cost estimates were presented in the local currency, Malaysian Ringgit (RM), whereby US $\$ 1=\mathrm{RM}$ 3.60 at the time of study.

\section{Patient population and clinical data collection}

PCI can be conducted as an emergency or elective procedure. Patients who undergo emergency PCI often have more severe comorbidities and disease presentation, leading to higher resource consumption and hospitalisation cost. They might develop complications post PCI, thus requiring admission to an intensive care unit. On the contrary, patients who undergo elective PCI have lower risk profile and likely fewer complications. As a relatively homogenous group, patients undergoing elective PCI would be a better proxy to analyse hospital-level cost. As such, we restricted our analysis to elective PCI, excluding patients with urgent/emergent indication for PCI, or with shock and haemodynamic instability. This definition is compatible with the definition of elective PCI adopted by other studies. ${ }^{12} 13$

\section{Study sites}

For the cost data collection, 13 public and private cardiac centres were invited to collaborate in this study. However, only five public cardiac centres agreed to participate. Being the tertiary referral hospitals, all centres are located in the most urbanised area of their respective region. They provide full-fledged cardiology services and is staffed by at least one interventional cardiologist. Centre I is a semicorporatised university teaching hospital, while centres II, III and IV are government public hospitals. Centre $\mathrm{V}$ is a specialised heart centre established as a corporatised entity. The range of centres was able to represent possible cost variations stemming from the heterogeneity in hospital characteristics, treatment preferences and geographical locations. Table 1 outlines the main description of each centre.

\section{Cost data collection}

Due to the inherent characteristics of financial and medical record keeping in each healthcare system, there is no pre-existing data collection tool suitable for use by all countries. For this study, our team of health economists and interventional cardiologists created a data collection tool using MS Excel, a program commonly used in local clinical and research facilities. Figure 1 outlines the steps of the cost data collection. For each centre, a data enumerator and a site coordinator were assigned. The data enumerator was in charge of the data collection from the respective departments. Site coordinators were senior personnel with vast knowledge on the daily clinical and financial operations of the centre, 
Table 1 Background information of study centres

\begin{tabular}{llllll}
\hline Centre & I & II & III & IV & V \\
\hline Ownership type & Semicorporatised & Public & Public & Public & Corporatised \\
Year of initiation of PCl service & 1987 & 1994 & 2011 & 2010 & 1992 \\
Interventional cardiologist* $^{*}$ & 5 & 3 & 1 & 1 & 15 \\
Fellow cardiologists* $^{*}$ & 12 & 8 & 7 & 0 & 12 \\
\hline Total hospital admission* & 34414 & 29336 & 1744 & 9340 & 8485 \\
\hline
\end{tabular}

*During the study period of January-June 2014.

$\mathrm{PCl}$, percutaneous coronary intervention.

especially pertaining to cardiology department and PCI procedures.

Pilot study was conducted at centre IV. Improvements were made based on shortcomings identified and feedbacks from data enumerator, site coordinator and data providers. Training workshops were organised to deliver topics such as the purpose of the study, definitions of cost items, introduction of the data collection tool and instructions on how to use the tool. On completion of data collection by data enumerator, site coordinator would check the completeness of the data and conduct face validity check to identify unjustifiable outliers. The compiled cost data was then forwarded to the primary investigator and assessed for its consistency and validity. Any unit cost outliers (defined as less than one-tenth or more than 10 times the average unit cost at all centres) were flagged and queried with the site coordinators. All the cost data were subsequently reviewed in the presence of cardiologists and deemed accurate and justifiable before further calculation and analysis.

\section{Costing pathway}

Step 1: identification of unit of analysis

To achieve the most accurate estimate of PCI cost, it is important to identify all the resources consumed by the patients(figure 2). For this study, we outlined the event pathway for the provision of care for PCI patients from admission to discharge. Two main units of analysis were identified, namely cardiac ward (CW) and cardiac catheterisation laboratory (CL). Based on guidelines in costing

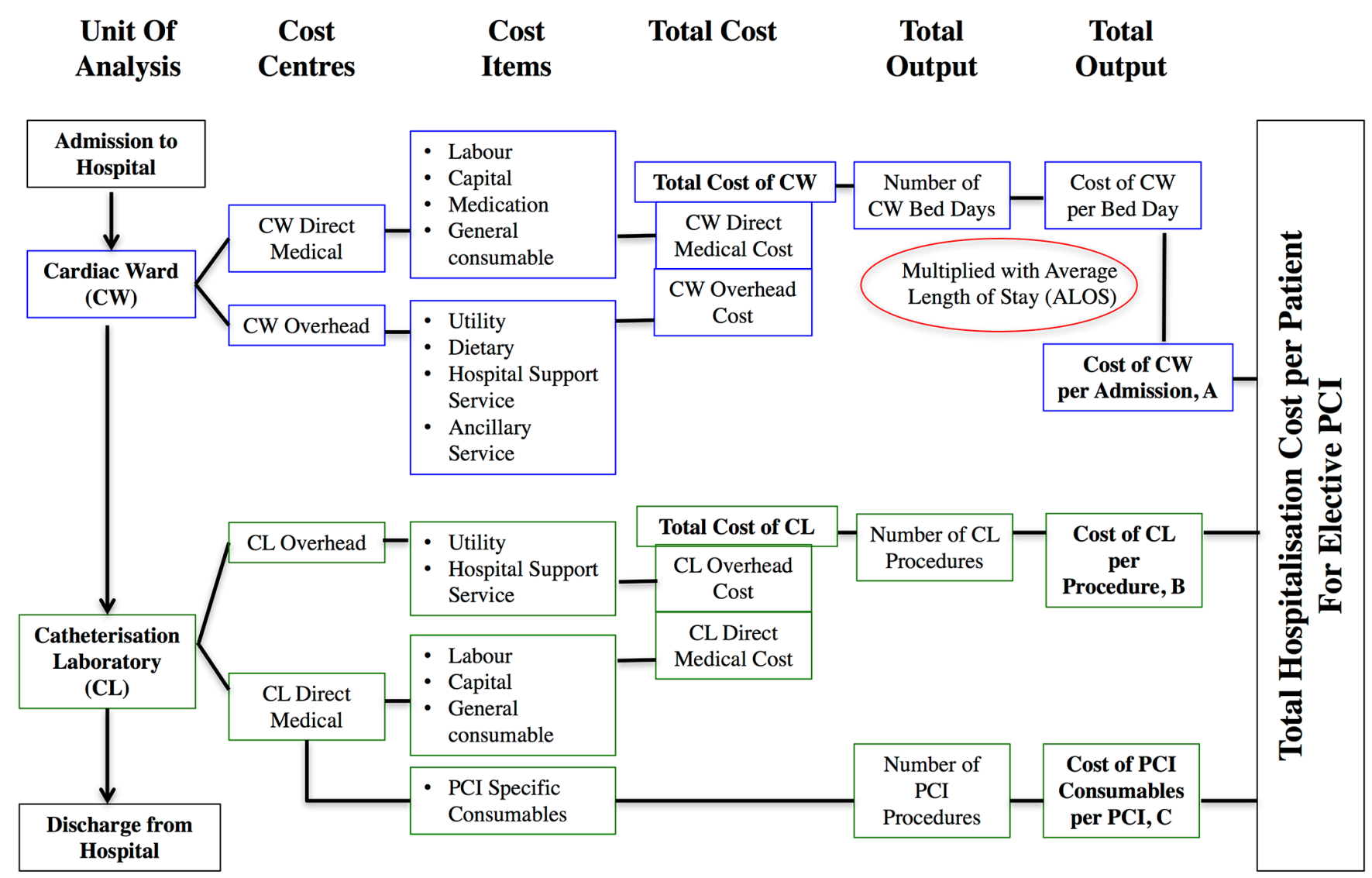

Figure 1 Pathway for the costing analysis of elective PCI. CL, catheterisation laboratory; PCl, percutaneous coronary intervention. 

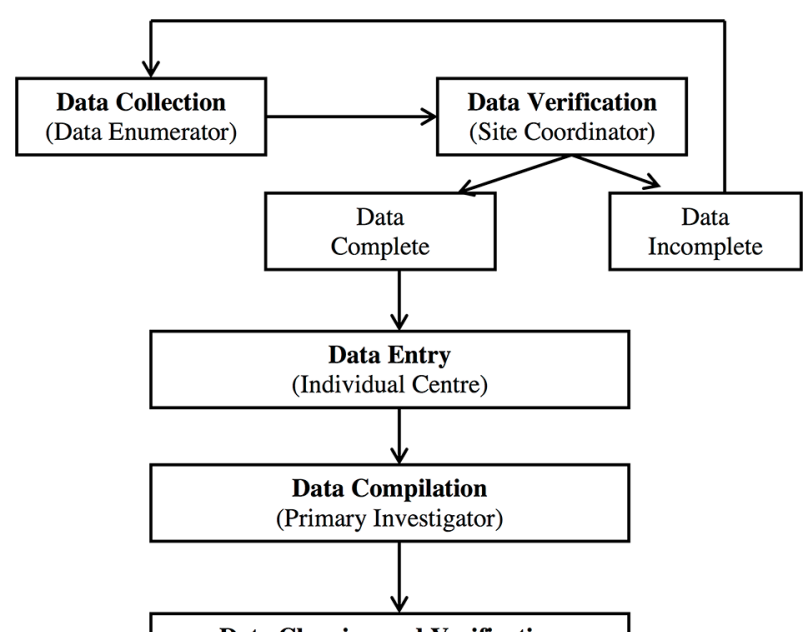

Data Cleaning and Verification

(Primary Investigator)

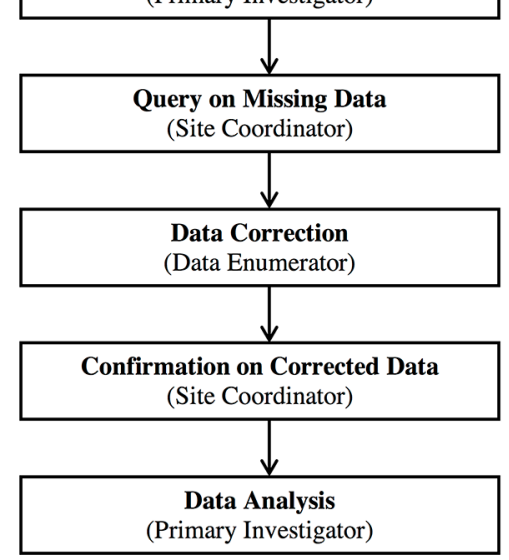

Figure 2 Flow chart of task and person-in-charge involved at each step of data collection process.

manual, health economists in our study team listed out various cost items that should ideally be included under each unit of analysis. The final list of cost items depended on general consensus of interventional cardiologists as they were the subject-matter experts. Under each unit of analysis, cost items that can be directly attributed to patient care are categorised under direct medical cost, while resources that are used by more than one department/unit in the hospital are categorised as overhead cost items.

\section{Step 2: identification of cost items}

For CW, direct medical cost items were labour, capital, consumables and medication, whereas overhead costs included utility, dietary, ancillary service and hospital support service. There were less cost items under CL, the second unit of analysis. Medications were prescribed and served at CW, thus not taken as a cost item under CL. Dietary and ancillary support service were also provided only once under CW.

\section{Step 3: valuation of cost items (table 2)}

As reference cost is still under development for public hospitals in Malaysia, actual calculation of unit cost for all the cost items was conducted using a top-down approach.
The subsequent sections discussed the valuation of each cost item.

\section{Labour}

This referred to the full salary inclusive of wages and employers' social contributions paid for the staff. A list of all the clinical staff involved in the provision of PCI service were used to obtain their payroll register from the finance department. For clinical staff stationed full time in the same unit, for example nurses and attendants in CW or laboratory technicians and radiographers in CL, their salaries were summed up in total as all of their productivity were contributed to their respective units. As for doctors, their work scope may spread across several units, including conducting procedures in the CL, treating patients in the specialist clinic and leading the ward rounds in CW. The interventional cardiologists in our study team were also the head of cardiology department at each centre. Therefore, their expert opinion was sought on the portion of time spent on various activities by doctors of different levels at their centre. The apportioned labour cost of doctors based on working hours assumed that all doctors were equally productive in delivering patient-related care and no idle time was spent.

\section{Capital}

This included fixed, one-time expenses incurred on the purchase of land, building, construction and equipment. As the main intention of this study was to obtain the operational cost of PCI procedure, land and building costs were excluded. Only equipment such as machine, furniture and medical instrument with cost higher than RM 1000 and economic useful life exceeding 1 year were included. The complete list of asset in CW and CL, together with their price and year of purchase were obtained from the inventory list. Using the straight-line depreciation approach, it was assumed that the services from the asset were divided equally over the useful life. A discount rate of $3 \%$ and a useful life of 5 years were used in conformity with most economic evaluations. ${ }^{14}$ The equivalent annual cost of each asset was calculated by annuitising the capital outlay using a discount rate with their respective useful life years.

\section{Consumables}

This included items that are disposable in nature and require regular replacement, such as syringes, cotton swabs and needles. The costs of general consumables were obtained from the procurement section of pharmacy department. For each of the cardiac centre, consumable provision may occur at different levels such as individual wards or departments. Depending on the best available cost information, workload ratio from the corresponding unit was used as the allocation basis to calculate the consumable cost. As for PCI-specific consumables such as cardiac stents, catheters, wires and balloons, the quantity and cost of purchase were retrieved from the purchase ledger. 


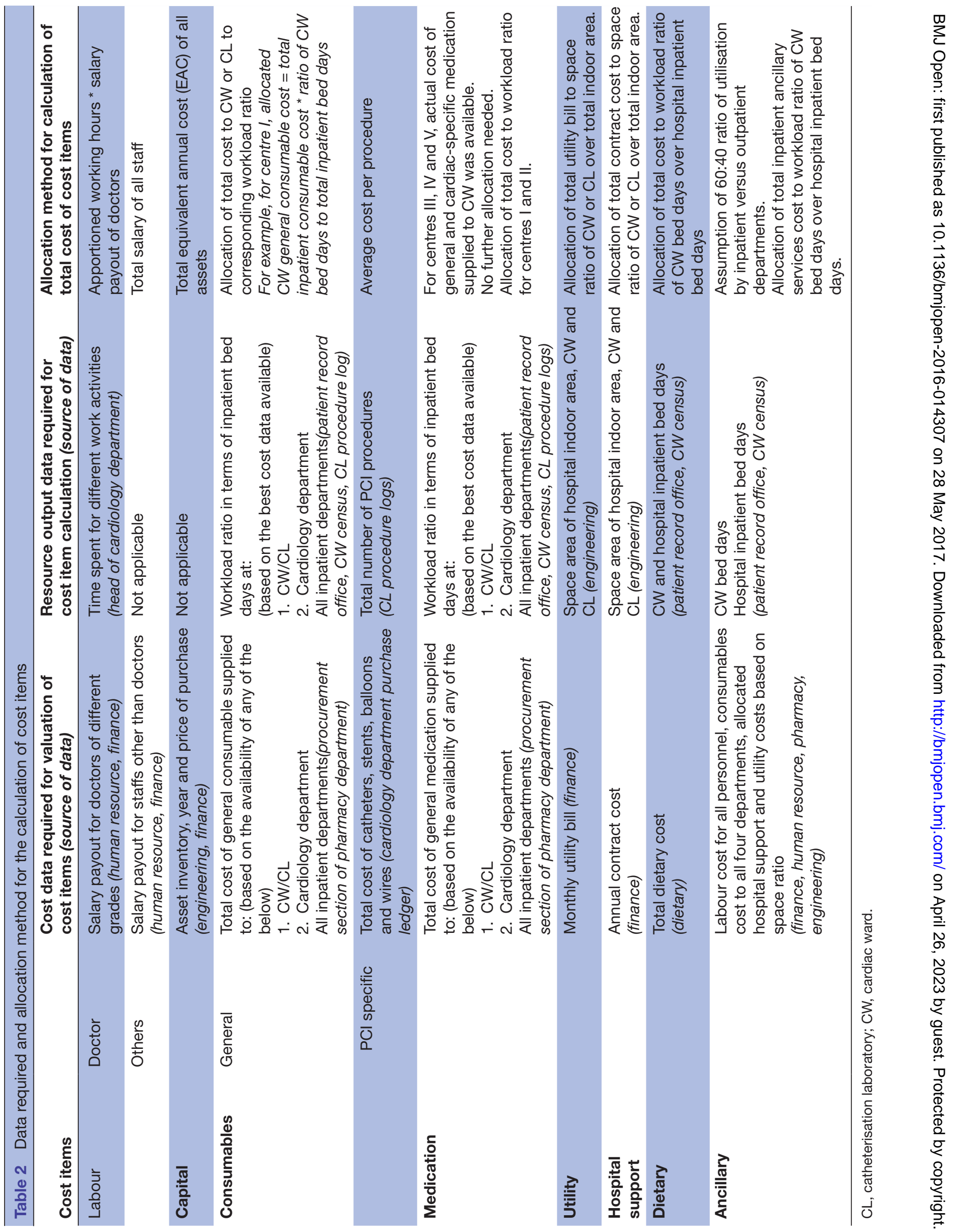




\section{Medication}

Under the drug purchasing mechanism in public hospitals, general medication and cardiac-specific medication were purchased by central pharmacy under separate budget. General medications are often stored as floor stock in the ward, whereas cardiac-specific medications would be distributed to cardiology department. Appropriate workload ratio was used as the allocation basis to determine the cost of general and cardiac-specific medication.

\section{Utility}

Utility cost referred to expenses incurred on electricity, water, telephone and Internet service. The total bill was obtained from the annual financial account. Hospital floor plan from the engineering department was obtained to calculate the space ratio, that is, the percentage of square metres of physical space occupied by the unit of analysis (CW or CL) in comparison with the hospital indoors area. Space ratio was the allocation basis to derive the utility cost of CW and CL.

\section{Hospital support service}

For all the participating centres, hospital support services such as housekeeping, laundry, waste management, building and equipment maintenance were outsourced as an annual contract to a private company. The annual contract cost was obtained from the finance department. Similar to utility cost, space ratio was used as the allocation basis.

\section{Dietary}

Cost of meal preparation by the in-house dietary unit was derived by allocating the total dietary cost to CW based on inpatient bed days.

\section{Ancillary service}

Services from pharmacy, radiology, laboratory and rehabilitation departments are usually provided to all hospital users. Due to time and budget constraints in this study, separate costing analysis was not conducted to assign the cost of each of these department to the individual patients. Instead, we followed the recommendation by Hendriks $e t$ $a l$ and grouped them as a context-specific overhead category, labelled as ancillary service. ${ }^{15}$ Its total cost would be the summation of the cost of general consumables supplied, the full salary pay-out for all the staff, the utility and hospital support services cost calculated in the same manner as mentioned above.

Based on a report in Malaysia, 60\% of clinical support services such as those provided by the ancillary departments in our study can be attributed to the inpatient use, compared with $40 \%$ for outpatient use. ${ }^{16}$ Therefore, the same 60:40 proportion was applied in this study for the cost of ancillary services. Using the workload ratio of CW to hospital inpatient bed days as allocation basis, the cost of ancillary service was calculated.
Step 4: calculation of unit cost

On valuation, the summation of all cost items gave rise to the total direct medical and overhead costs incurred at each unit of analysis. To delineate the cost incurred by patients who underwent elective PCI, suitable denominators were applied. For CW, the inpatient bed day was deemed as an objective indicator for the workload. By dividing the total cost of CW with the number of CW inpatient bed days during the study period, we were able to produce average cost per bed day. The cost per bed day was further multiplied by an average length of stay (ALOS), giving rise to the average cost per admission. The ALOS for an elective PCI patient was decided to be 3 days via consensus of all the interventional cardiologists in the study. For centre III, the weighted admission cost was calculated based on the ratio of elective PCI patients admitted to inpatient ward and day care.

For CL, the second unit of analysis, the number of CL procedures conducted was used as the denominator to derive the cost per CL utilisation. As PCI consumables were not shared by all procedures conducted in CL, we applied a separate denominator, that is, the number of PCI procedures, to calculate the PCI consumables cost per procedure. Together, this gave rise to cost per PCI procedure in CL.

The summation of cost per admission and cost of per PCI procedures in CL led to the total hospitalisation cost of a patient who underwent elective PCI.

\section{RESULTS}

Based on the methods described, a range of results can be generated (table 3). Comparisons can be made at the levels of cost item, unit of analysis (CW or CL), up to the total cost. It is important to note that the cost was produced via a top-down approach, and it represented the average cost estimates for a patient admitted for an elective PCI procedure, assuming ALOS of 3 days, with no complications post PCI that required intensive care. This cost also did not reflect any difference in the type and number of stents used for each patient.

The total hospitalisation cost ranged from RM11 471 (US\$3186) to RM14 465 (US\$4018). The CW admission cost accounted for one-fifth or less of the total hospitalisation cost at all centres. On the contrary, cost incurred at CL was four times that of the admission cost. PCI consumables contributed to the biggest proportion among all the cost items, accounting for more than half of the total costs.

Total hospitalisation cost was the highest at centre II. Closer scrutiny revealed that average cost of PCI consumables of centre II and its proportion over the total hospitalisation cost were highest across the five centres. In contrast, centre III, the centre with a day care service for PCI patients, recorded the lowest cost of CW admission. This partly contributed to cheapest hospitalisation cost in centre III, at $12.3 \%$ to $26.1 \%$ lower compared with the other centres. Direct medical cost items were generally 


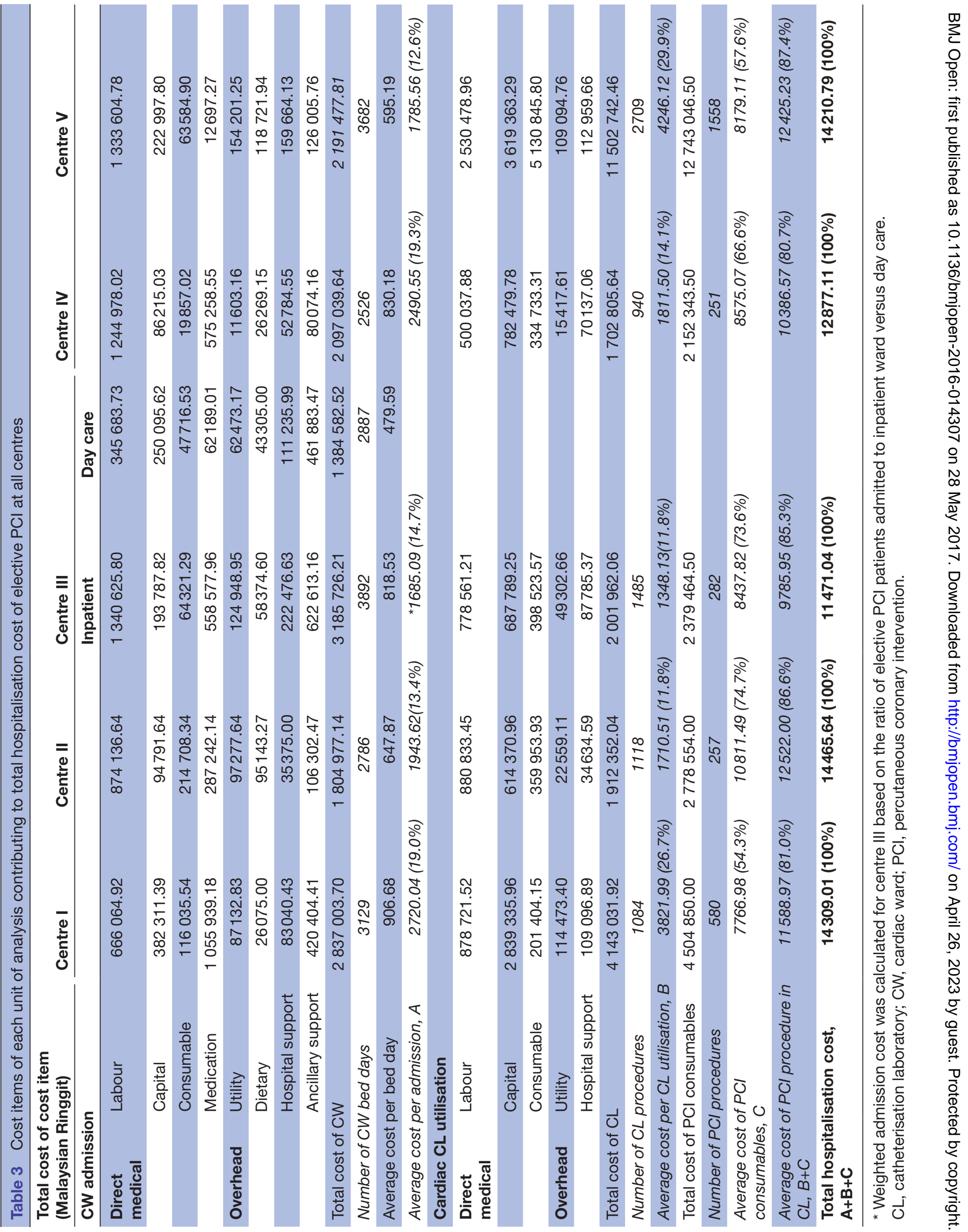


more expensive than overhead cost items in both CW and CL. For CW admission, medication and labour costs were the highest. As for CL utilisation, capital cost ranked the highest at centres I and V, the two corporatised hospitals.

\section{DISCUSSION}

As CVD represents the leading cause of mortality and morbidity in LMIC, expansion of the cardiology service is inevitable. However, most LMIC lack guidance for budget and resource planning as they do not have any official economic evaluation guidelines, and the international guidelines are not a good fit for LMIC. There is also a lack of infrastructure support to sustain a comprehensive health informatics data. These are indispensable components to produce reliable cost-related outcome evidence towards better policy making. In Malaysia, there is a lack of easily accessible information such as accurate unit costs, detailed financial account and complete patient records, all of which are vital to conduct economic evaluation. In this paper, we outlined the study design of a multicentre costing analysis for elective PCI, based on the limitations in our local setting, before presenting the cost output. Overall, the results demonstrated substantial costs associated with the provision of PCI, with PCI consumables being the dominant cost item across all centres.

In the course of designing the costing analysis, we made some modifications to previously published guidelines. International guidelines recommended the design of a standardised cost collection tool that is universally available and easy to use for all involved parties. ${ }^{17} 18$ Thus, we chose MS Excel over other more sophisticated costing software, in view of its wide accessibility and user-friendliness. Many HIC used Diagnosis Related Group, a system that classifies acute inpatient episodes based on the main clinical condition, as unit of analysis for illness-specific costing. ${ }^{19}$ However, this is not a common practice in our local hospitals. To overcome this, we used an event pathway from admission to discharge to identify cost items under the respective units of analysis. Furthermore, reference cost from national database of HIC are available for cost item valuation, such as in the UK and Australia. ${ }^{20}{ }^{21}$ In the absence of reference cost, certain literature proposed the application of cost-to-charge ratio, which is a relatively simpler and less time-consuming method to reflect the actual cost. ${ }^{22}$ However, it would be a suboptimal cost estimate as public healthcare is highly subsidised in Malaysia and the charges would be a poor proxy for the actual cost incurred. As a result, we conducted actual calculation of unit cost with top-down approach. Between top-down and bottom-up approaches, there is no consensus as to which is the gold standard. Typically, top-down approach is more straightforward but the trade-off is a lack of sensitivity, especially for cost items that originated from a less homogenous production. On the contrary, bottom-up approach is able to produce a more accurate cost estimate, but the main limitation lies within the complexity of its implementation, especially across different study centres. ${ }^{23}$ Several authors advocated bottom-up approach but acknowledged that such data may not be easily available in LMIC. ${ }^{3425}$ As this costing study involved five centres, a full top-down approach was applied to standardise the cost data collection in order to produce reliable cost estimates for comparison between the centres.

The application of economic evaluation in routine clinical practice is not widespread in Malaysia. The lack of familiarity among the staff in healthcare facilities towards the conduct of costing analysis was a major barrier in our study. All private hospitals cited confidentiality issue of financial data sharing as reason of non-participation. During data collection, there were circumstances of bureaucratic resistance towards parting with cost data of sensitive nature. Misperception of the costing exercise as a performance audit was another reason behind some of the staff reluctance to cooperate. As a result, the relationship between the enumerators and other hospital staff proved to be a vital factor towards a successful data collection. In several instances, site coordinators had to step in and used their discretion to ensure that complete data were provided. This highlighted the importance of ensuring access of transparent and quality cost data towards the successful implementation of a costing analysis.

There were several limitations. Selection bias existed, as there was no input from private for-profit healthcare centres. Despite this, the cost output from this study would be of tremendous value as the major bulk of care burden in Malaysia fell on the public healthcare system. This is reflected in a national survey that showed $65.0 \%$ of the Malaysian population sought treatment at public healthcare facilities and $52.4 \%$ of the total health expenditure was incurred in the public sector. ${ }^{26} 27$ The exclusion of land and building costs may reduce the applicability of capital cost findings to rural, district hospitals. However, this has less impact towards costing of highly specialised medical procedures such as PCI, as the provision of such services in Malaysia will likely remain in tertiary-level hospitals in urban locations. Another limitation being the potential bias arising from inherent differences in the financial record keeping between the centres. This may not be completely eliminated despite our best effort to standardise the data collection process, as shown in the derivation of consumable and medication costs. Despite the shortcomings, the key strengths of this study lie in the practical applications of its methodological contribution and cost findings. We shared the alternative pathways we devised to overcome barriers in conducting a costing analysis in an LMIC setting. To the best of our knowledge, this is the first multicentre costing analysis of cardiac service in Malaysia. Average cost estimates derived from five cardiac centres with different ownership and administration characteristics enabled us to compare the resource utilisation and cost output at various levels of the costing pathway. 
While direct cost comparison with other published literatures would not be completely feasible owing to the heterogeneous study populations, different perspectives used in cost estimation, varying cost calculation methods and vastly different healthcare systems, our finding of PCI consumables being the dominant cost item strikes a similar chord with many studies. ${ }^{28-30}$ This shows the importance of an efficient consumable purchasing mechanism, especially for cardiac stents. Policy makers should review the current procurement practice of PCI consumables by individual cardiac centres. Vigorous negotiation via competitive bidding processes, ideally handled via a central purchasing agency, may lead to potential cost reduction. Furthermore, results from one of the study centres showed that day care establishment can be an attractive cost-saving strategy. The general consensus among relevant studies found that low-risk patients were good candidates for same-day discharge after uncomplicated PCI. ${ }^{31}{ }^{32}$ Nevertheless, further research in more Malaysian hospitals are warranted to study the feasibility of establishing large-scale day care service for cardiac patients.

Overall, the corporatisation and teaching status of hospitals in this study appear to have minor influence to the eventual cost. However, the cost differences may also be due to the casemix and outcome of patients at each centre. Further casemix-adjusted analysis could be conducted to analyse the factors that contribute to the difference in the cost output. In addition, results from this study can be incorporated with clinical data to facilitate individual-level patient analysis, such as the identification of cost predictors via regression analysis. The unit cost derived from the various levels of the care pathway can also be applied to economic evaluation such as cost-effectiveness analysis or economic modelling in relevant research. All these will provide valuable information to the cardiac centres and policy makers for proper resource and budget allocation. Lastly, while in-hospital treatment may account for a substantial portion of overall cost of CAD care, pre-PCI and post-PCI cost to the healthcare providers, patients and society may represent hidden economic burden. This is an important area for future research.

\section{CONCLUSION}

The CVD epidemic in LMIC calls for targeted interventions from all aspects of healthcare delivery. Currently, the application of evidence from economic evaluation for health policy decision making in Malaysia is very limited due to the obstacles in conducting costing studies. We devised alternative costing pathways to overcome the barriers and conducted a multicentre costing analysis for elective PCI. The findings highlighted the need for effective procurement practice in view of the high cost of PCI consumables. Shorter hospitalisation stay via daycare establishment represents another potential cost-saving mechanism. Future studies can be built on our efforts for other medical procedures or healthcare service. Recommendations from this study would also be useful for like-minded researchers who wish to conduct similar costing studies in LMIC.

\section{Author affiliations}

${ }^{1}$ Department of Social and Preventive Medicine, Faculty of Medicine, University of Malaya, Kuala Lumpur, Malaysia

${ }^{2}$ Department of Cardiology, Sarawak Heart Centre, Sarawak, Malaysia

${ }^{3}$ Pharmaceutical Services Division, Ministry of Health, Petaling Jaya, Malaysia ${ }^{4}$ Department of Pharmacy, Clinical Research Centre, Queen Elizabeth 2 Hospital, Kota Kinabalu, Malaysia

${ }^{5}$ Department of Pharmacy, Sarawak Heart Centre, Sarawak, Malaysia ${ }^{6}$ Administrative Office, Penang General Hospital, Pulau Pinang, Malaysia ${ }^{7}$ Division of Cardiology, Clinical Research Centre, Queen Elizabeth 2 Hospital, Kota Kinabalu, Malaysia

${ }^{8}$ Department of Cardiology, National Heart Institute, Kuala Lumpur, Malaysia

${ }^{9}$ Division of Cardiology, Penang General Hospital, Penang, Malaysia

${ }^{10}$ Division of Cardiology, Department of Medicine, Faculty of Medicine, University of Malaya, Kuala Lumpur, Malaysia

${ }^{11}$ Department of Social and Preventive Medicine, Faculty of Medicine, University of Malaya, Julius Centre University of Malaya, 50603 Kuala Lumpur, Malaysia

Correction notice This paper has been amended since it was published Online First. Owing to a scripting error, some of the publisher names in the references were replaced with 'BMJ Publishing Group'. This only affected the full text version, not the PDF. We have since corrected these errors and the correct publishers have been inserted into the references.

Acknowledgements The authors would like to thank all cardiologists and nurses at each participating centres for their effort in data collection.

Contributors OI, MD, HBL, TKO, RMA, WAWA, SYL, EVL and KYL conceived the overall study. All authors are involved in the design of the study. WAWA, OI, HBL, TKO and RMA led the implementation of the study at each centre, while KYL, EVL, SYL, LA and SH coordinated the data collection. KYL drafted the manuscript. OTK, MAS and MD revised the manuscript critically for intellectual content. All authors read and approved the final manuscript.

Funding This work was supported by the Malaysia Ministry of Higher Education High Impact Research MoE Grant UM.C/625/1/HIR/MoE/ E000010-20001 and Malaysia Ministry of Health MRG grant (MRG-MOH-2014-02).

Competing interests None declared.

Patient consent Not obtained.

Ethics approval This study is approved by the Malaysian Ministry of Health Medical Research and Ethics Committee (ID: NMRR-13-1403-18234 IIR).

Provenance and peer review Not commissioned; externally peer reviewed. Data sharing statement № additional data are available.

Open Access This is an Open Access article distributed in accordance with the Creative Commons Attribution Non Commercial (CC BY-NC 4.0) license, which permits others to distribute, remix, adapt, build upon this work non-commercially, and license their derivative works on different terms, provided the original work is properly cited and the use is non-commercial. See: http://creativecommons.org/ licenses/by-nc/4.0/

(C) Article author(s) (or their employer(s) unless otherwise stated in the text of the article) 2017. All rights reserved. No commercial use is permitted unless otherwise expressly granted.

\section{REFERENCES}

1. Drummond M, Sculpher M, Torrance G, et al. Methods for the economic evaluation of health care programmes. 3rd ed. Oxford: Oxford University Press, 2005.

2. Lipscomb J, Yabroff KR, Brown ML, et al. Health care costing: data, methods, current applications. Med Care 2009;47:S1-S6.

3. Mogyorosy Z, Smith PC. The main methodological issues in costing health care services: a literature review. Centre for Health Economics, University of York, 2005, http://www.york.ac.uk/media/che/ documents/papers/researchpapers/rp7_Methodological_issues_in_ costing_health_care_services.pdf. 
4. von Both C, Jahn A, Fleba S. Costing maternal health services in South Tanzania: a case study from Mtwara Urban District. Eur J Health Econ 2008;9:103-15.

5. Sarowar MG, Medin E, Gazi R, et al. Calculation of costs of pregnancy- and puerperium-related care: experience from a hospital in a low-income country. $J$ Health Popul Nutr 2010;28:264-72.

6. Briggs A, Nugent R. Editorial. Health Econ 2016;25(Suppl 1):6-8.

7. Planning and Development Division HIC. Health Facts 2013. Malaysia;2014 http://www.moh.gov.my/images/gallery/publications/ HEALTH FACTS 2014.pdf.

8. Keeley EC, Boura JA, Grines CL. Primary angioplasty versus intravenous thrombolytic therapy for acute myocardial infarction: a quantitative review of 23 randomised trials. Lancet 2003;361:13-20.

9. Van de Werf F. Drug-eluting stents in acute myocardial infarction. $N$ Engl J Med 2006;355:1169-70.

10. Evans E, Imanaka Y, Sekimoto M, et al. Risk adjusted resource utilization for AMI patients treated in Japanese hospitals. Health Econ 2007;16:347-59.

11. Polanczyk CA, Lane A, Coburn M, et al. Hospital outcomes in major teaching, minor teaching, and nonteaching hospitals in New York state. Am J Med 2002;112:255-61.

12. Negassa A, Monrad ES. Prediction of length of stay following elective percutaneous coronary intervention. ISRN Surg 2011;2011:1-6.

13. Shaw RE, Anderson HV, Brindis RG, et al. Development of a risk adjustment mortality model using the American College of Cardiology-National Cardiovascular Data Registry (ACC-NCDR) experience: 1998-2000. J Am Coll Cardiol 2002;39:1104-12.

14. Creese A, Parker D. Cost analysis in primary health care: a training manual for programme managers. Geneva: World Health Organization, 2000.

15. Hendriks ME, Kundu P, Boers AC, et al. Step-by-step guideline for disease-specific costing studies in low- and middle-income countries: a mixed methodology. Glob Health Action 2014;7:23573.

16. Division MD. Guideline on cost data Collection for the Casemix System. Malaysian DRG 2013.

17. Batura N, Pulkki-Brännström AM, Agrawal P, et al. Collecting and analysing cost data for complex public health trials: reflections on practice. Glob Health Action 2014;7:23257.

18. Drummond $M$, Brandt $A$, Luce $B$, et al. Standardizing methodologies for economic evaluation in health care. practice, problems, and potential. Int J Technol Assess Health Care 1993;9:26-36.

19. Tan SS, Serde' $\mathrm{n}$, van Ineveld BM, et al. DRGs and cost accounting: which is driving which? Diagnosis-related groups in Europe: towards efficiency and quality. Berlin: Open University Press, 2011.
20. NHS reference costs 2014 to 2015. United Kingdom, 2015. https:// www.gov.uk/government/publications/nhs-reference-costs-2014-to2015.

21. Pharmaceutical Benefits Advisory Committee. Manual of resource items and their associated costs. Canberra, Australia, 2009. http:// www.health.gov.au/internet/main/publishing.nsf/Content/health-pbsgeneral-pubs-Manual-content.htm-copy2.

22. Shwartz M, Young DW, Siegrist R. The ratio of costs to charges: how good a basis for estimating costs? Inquiry 1995;32:476-81.

23. Emmett $D$, Forget $R$. The utilization of activity-based cost accounting in hospitals. J Hosp Mark Public Relations 2005;15:79-89.

24. Tan SS, Rutten FF, van Ineveld BM, et al. Comparing methodologies for the cost estimation of hospital services. Eur J Health Econ 2009;10:39-45.

25. Clement Nee Shrive FM, Ghali WA, Donaldson C, et al. The impact of using different costing methods on the results of an economic evaluation of cardiac care: microcosting vs gross-costing approaches. Health Econ 2009;18:377-88.

26. Malaysia National Health Accounts Unit. Malaysia National Health Accounts Health Expediture Report 1997-2012. Malaysia 2014.

27. Institute for Public Health. The Third National Health and Morbidity Survey (NHMS III) 2006. Kuala Lumpur, 2008.

28. Varani E, Balducelli M, Vecchi G, et al. Comparison of multiple drug-eluting stent percutaneous coronary intervention and surgical revascularization in patients with multivessel coronary artery disease: one-year clinical results and total treatment costs. J Invasive Cardiol 2007;19:469-75

29. Manari A, Costa E, Scivales A, et al. Economic appraisal of the angioplasty procedures performed in 2004 in a high-volume diagnostic and interventional cardiology unit. J Cardiovasc Med 2007;8:792-8.

30. Chino M, Sakamoto M, Sasaki T, et al. [What aspects are inefficient concerning percutaneous coronary intervention in Japan?: international cost-effectiveness comparisons]. J Cardiol 2004;44:85-92.

31. Abdelaal E, Rao SV, Gilchrist IC, et al. Same-day discharge compared with overnight hospitalization after uncomplicated percutaneous coronary intervention: a systematic review and metaanalysis. JACC Cardiovasc Interv 2013;6:99-112

32. Brayton KM, Patel VG, Stave C, et al. Same-day discharge after percutaneous coronary intervention: a meta-analysis. J Am Coll Cardiol 2013;62:275-85. 